\title{
A simple method to produce 2D and 3D microfluidic paper-based analytical devices for clinical analysis
}

\author{
Ricardo A.G. de Oliveira ${ }^{\text {, }}$, Fiamma Camargo ${ }^{\text {a }}$, Naira C. Pesquero ${ }^{\mathrm{b}}$, Ronaldo Censi Faria ${ }^{\text {a, * }}$ \\ a Departamento de Química, Universidade Federal de São Carlos, São Carlos, SP, 13565-905, Brazil \\ ${ }^{\mathrm{b}}$ Intituto de Química-UNESP, Av. Francisco Degni s/n, Araraquara, SP, 14800-900, Brazil
}

\section{H I G H L I G H T S}

- An easily and fast procedure to produce sealed $\mu$ PADs is proposed.

- $\mu$ PADs are fabricated using low-cost materials and equipment.

- Hundreds of 2D and 3D $\mu$ PADs can be produces rapidly.

- $\mu$ PADs have been successfully applied in blood and urine analysis.

\section{A R T I C L E I N F O}

\section{Article history:}

Received 16 September 2016

Received in revised form

19 December 2016

Accepted 2 January 2017

Available online 5 January 2017

\section{Keywords:}

Paper-based analytical devices

Low-cost diagnostics

Point-of-care devices

Colorimetric detection

\section{G R A P H I C A L A B S T R A C T}

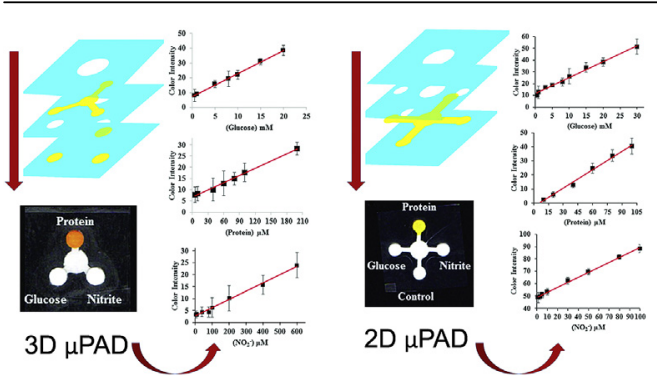

\begin{abstract}
A B S T R A C T
This paper describes the fabrication of 2D and 3D microfluidic paper-based analytical devices ( $\mu$ PADs) for monitoring glucose, total protein, and nitrite in blood serum and artificial urine. A new method of cutting and sealing filter paper to construct $\mu$ PADs was demonstrated. Using an inexpensive home cutter printer soft cellulose-based filter paper was easily and precisely cut to produce pattern hydrophilic microchannels. 2D and 3D $\mu$ PADs were designed with three detection zones each for the colorimetric detection of the analytes. A small volume of samples was added to the $\mu$ PADs, which was photographed after 15 min using a digital camera. Both $\mu$ PADs presented an excellent analytical performance for all analytes. The 2D device was applied in artificial urine samples and reached limits of detection (LODs) of $0.54 \mathrm{mM}$, $5.19 \mu \mathrm{M}$, and $2.34 \mu \mathrm{M}$ for glucose, protein, and nitrite, respectively. The corresponding LODs of the 3D device applied for detecting the same analytes in artificial blood serum were $0.44 \mathrm{mM}, 1.26 \mu \mathrm{M}$, and $4.35 \mu \mathrm{M}$.
\end{abstract}

() 2017 Elsevier B.V. All rights reserved.

\section{Introduction}

In recent years, the interest in the development of microfluidic paper-based analytical devices ( $\mu$ PADs) has grown exponentially $[1,2]$. $\mu$ PADs are a new class of point-of-care devices, which combine the capabilities of conventional microfluidic devices with

\footnotetext{
* Corresponding author.

E-mail address: rcfaria@ufscar.br (R.C. Faria).
}

simple tests on paper strips. Aspects such as low cost, easy use, low sample consumption, simple fabrication, and high potential for use in remote locations are some of the main reasons behind the interest in $\mu$ PADs. They have been applied as accessible tools for chemical, biological, or clinical analyses in developing countries or in remote areas as well as in emergency situations [1,3,4]. The advantage of using $\mu$ PADs is the possibility of handling small sample volumes, thereby saving valuable reagents, reducing cost per analysis and providing rapid diagnosis requiring no trained personnel [5]. 
The use of cellulose paper as a substrate for creating $\mu$ PADs presents several advantages including: (i) it is thin and light $(\sim 10 \mathrm{mg} /$ $\left.\mathrm{cm}^{2}\right)$, is available in a wide range of thicknesses $(0.25-1.50 \mathrm{~mm})[5]$, and is easy to storage and transport; (ii) biocompatibility with biological samples [6]; (iii) paper can be easily printed, coated, modified, and cut [6,7]; (iv) it is biodegradable or can be easily burned, what is the ideal case for safe single usages [6]; (v) cellulose paper is inexpensive (US\$ 0.15/ $\mathrm{m}^{2}$-US\$ $1.20 / \mathrm{m}^{2}$ ) [6]; and (vi) it can be used for sample pretreatment, since filter papers with well-defined pores can separate suspended solids from samples [1,6]. $\mu$ PADs have received enormous attention in recent years with promising applications in immunoassays [8], urine [9], and saliva analysis [10], environmental monitoring [11,12], and blood tests, using colorimetric [11,13,14], electrochemical [15-17], chemiluminescent $[8,18]$, and other detection methods [19].

Photolithography [20] and wax printing $[9,21,22]$ are among the main techniques used for fabricating $\mu$ PADs [23-25]. Other techniques for creating hydrophilic channels in paper include the use of desktop plotter to print [26], stamp [27] or draw using commercial and in-house inks [28], poly(dimethylsiloxane) (PDMS), plasma treatment of hydrophobized paper [29,30] laser cutting [31], and printer cutting [32,33]. These manufacturing techniques still present many drawbacks for production of $\mu$ PADs at large scale, including the use of expensive equipment, as those used in photolithography or the $\mathrm{CO}_{2}$ laser for cutting, as well as, the needed of too many steps as, e.g. the washing cycles to remove noncrosslinked polymer in the photolithography method [33]. Clipping methods involving nitrocellulose [33] or glass fiber membranes [34], which are harder and more expensive than usual cellulose-based filter paper, have been suitable for fabricating $\mu$ PADs. Recently, it was reported that cellulose-based filter paper cannot be properly cut using knife cutters [34]. Yet Fenton et al. fabricated 2D lateral-flow devices cut from cellulose paper or nitrocellulose membrane previously surrounded by a polyester tape using a "kiss cuts" procedure, which was based on three sequential overlapping cuts with adjustment of knife height to gradually increase cutting deep, however, the costly nitrocellulose membrane was preferentially used [33]. Cassano and Fan developed 2D $\mu$ PADs based on chromatographic paper cut using a digital craft cutter and thermal roll laminator to seal the devices [32]. To avoid tearing, the paper was covered with a sacrificial polyester film and cut in two steps using a high-force-cutting. Although the cellulose paper was cut without tearing using high-force-cutting, this cut mode cannot be found in low-cost home cutter printer.

In this paper, we describe an alternative method for fabricating 2D and 3D $\mu$ PADs platforms based on a simple procedure to cut soft cellulose filter paper with an inexpensive home cutter printer and plastic adhesives to seal the microfluidic devices, thereby allowing ease and safe handling and transportation of the $\mu$ PADs. The 2D and 3D $\mu$ PADs produced were evaluated in bioassays applications encompassing colorimetric detection of glucose, nitrite, and total protein in simulated blood serum and urine.

\section{Experimental}

\subsection{Chemicals and materials}

(+) Glucose (99.5\%), glucose oxidase (from Aspergillus Niger, $215 \mathrm{U} \mathrm{mg}^{-1}$ ), peroxidase (from horseradish, $113 \mathrm{U} \mathrm{mg}^{-1}$ ), potassium iodate, bovine serum albumin (98\%), bromophenol blue, potassium chloride, citric acid, sodium citrate, sodium nitrite, n-(1-napthyl) ethylenediamine, and sulphanilamide were purchased from SigmaAldrich (St. Louis, MO). Sodium carbonate, sodium bicarbonate, sodium phosphate dibasic, magnesium chloride, sodium chloride, urea, calcium chloride, magnesium sulphate, sodium sulphate, potassium phosphate monobasic, potassium phosphate dibasic, and ammonium chloride were purchased from J.T. Baker (Xalostoc, MEX). Lactic acid and hydrochloric acid were purchased from Synth (S. Paulo, Brazil). Vinyl adhesive, laminating pouch, and laser printer transparency film were acquired in local market.

\subsection{Instrumentation}

RGB images of the $\mu$ PADs were acquired in JPEG format using a simple compact Cybershot DSC-W730 camera (Sony). The images were converted to CMYK or grayscale format using the Corel PHOTO-PAINT X7 software, by selecting the relevant image area and calculating the average color intensity. All $\mu$ PADs were photographed inside a black box mounted with four internal white LEDs, as previously described [35]. Briefly, the system consisted of a black plastic box with an aperture at its top, wherein the digital camera lens was positioned. Four ultra-bright white LEDs powered with $12 \mathrm{~V}$ internal batteries were used for keeping a continuous light intensity within the box. The internal compartment of the system was painted in matt black to eliminate reflection effects. The $\mu$ PADs were placed at the bottom of the system, thus avoiding reflections from the LEDs' light. Pictures were then taken with the system completely closed using the digital camera in auto-exposure mode. Optical microscopy images were obtained using a B\&W Tek microscope running with a $40 \times$ objective lens.

\subsection{Fabrication of $\mu P A D s$}

2D and 3D $\mu$ PADs were fabricated by drawing patterns on paper and vinyl mask using a home cutter printer (Silhouette Cameo), which was controlled by the Silhouette Studio software. The software allows to choose between continuous and dotted cut. The cutter printer was used to cut different materials to fabricate the $\mu$ PADs, namely, cellulose filter paper (Whatman Chr 1), vinyl adhesive, and laminating pouches purchased in local market. The $\mu$ PADs were pressed and sealed using a locally purchased heat press machine.

\subsection{Colorimetric assay and solution}

The protocols of standards and simulated urine and blood serum solutions as well as the glucose, total protein, and nitrite assays are described in the Supporting Information (SI).

\section{Results and discussion}

\subsection{Fabrication of $\mu P A D s$}

One of the major challenges to produce $\mu$ PADs is developing a simplest procedure capable of creating hydrophilic microfluidic channels in inexpensive cellulose-paper substrates, which could be easily applied for large scale production. A series of techniques have been used to overcome this challenge, e.g., photolithography, wax printing, PDMS stamp, and knife and laser cutting. Regarding the use of knife cutters, some authors have described the difficulty to cut filter papers. Harder and costly materials including nitrocellulose paper and glass fiber membrane have been preferentially used to fabricate $\mu$ PADs because they are easier to cut by knifes $[33,36]$. Using an inexpensive home cutter printer $(\sim 300)$, we were able to cut soft cellulose-based filter paper in two simple steps: first the paper was covered with a vinyl adhesive on one side. The vinyl adhesive was a commercially available tape, which could be easily detached from the paper without removing cellulose fibers and leaving no residue. Second, the paper was cut on the cutter printer using a "perforation cutting" procedure followed by overlapped 
continuous cutting. The "perforation cutting" was carried out chosen a dotted cut mode in the software of the cutter printer. If this mode of cut is not available in the cutter printer the perforation cutting can be achieved by drawing the pattern of the $\mu$ PAD using dotted lines. Dotted lines with shorter and closer dots will give the best results in terms of the quality of the cut. Fig. 1(A and B) shows a piece of filter paper sheet cut into the microfluidic device pattern and, as can be seen, the use of perforation cutting allowed cut the paper into patterns with different sizes with good precision. On the other hand, as previously reported, when a continuous cutting was performed, tears and ruptures were formed, which did not allow cutting the paper correctly, as illustrated in Fig. 1C. The perforation cutting allows to cut soft cellulose based chromatographic paper to form channels with thickness as small as $0.3 \mathrm{~mm}$ and spots with minimum diameters of $0.5 \mathrm{~mm}$ (see Fig. S1). Hence, the use of perforation cutting followed by continuous cutting without knife height variation allowed cutting filter paper perfectly into any desired pattern and size. The perforation cutting method was then applied to fabricate patterned $2 \mathrm{D}$ and $3 \mathrm{D} \mu \mathrm{PADs}$, as follows:

\subsection{Fabrication of $2 D \mu P A D s$}

Initially, a thermal laminating pouch was marked with 5 holes,

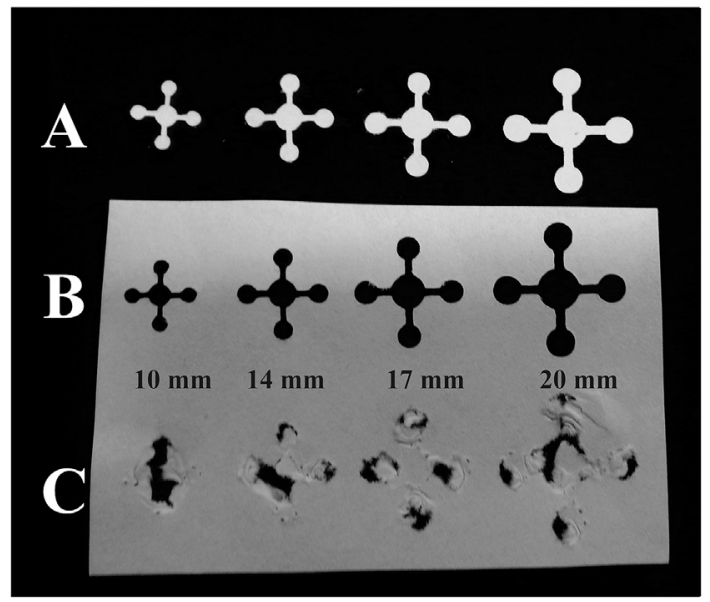

Fig. 1. Filter paper cut into microfluidic device patterns with different sizes $(10,14,17$, and $20 \mathrm{~mm}$ ) (A). Performance difference between perforation cutting (B) and continuous cutting $(\mathrm{C})$ on a same filter paper sheet. one at the middle $(\mathrm{r}=3.25 \mathrm{~mm})$ to be used as an inlet for sample addition and $4(\mathrm{r}=2.00 \mathrm{~mm})$ distanced from that central hole in a cross-shaped position and used for adding reagents to storage in paper spot zone, Fig. 2A-b. The filter paper was then cut into the same cross shape using the perforation cutting procedure with 4 detection zone spots at the corners which were connected to the central spot used as the inlet for sample solutions ( $16.00 \mathrm{~mm} \times 16.00 \mathrm{~mm}$ ), Fig. $2 \mathrm{~A}-\mathrm{c}$. The $2 \mathrm{D} \mu \mathrm{PAD}$ was fabricated by mounting the patterned paper between a polyester sheet (laser printer transparency film) and the laminating pouch in such way that its thermal adhesive face contacted the paper and its 5 holes matched the 5 paper spots (total dimension of $20.66 \mathrm{~mm} \times 20.66 \mathrm{~mm}$ ). Once assembled, the $\mu$ PAD was heated at $120^{\circ} \mathrm{C}$ and pressed for $120 \mathrm{~s}$ on a heat press machine for sealing. Afterwards, the reactant solutions for colorimetric bioassays were added to the paper spots using the 4 holes made on the laminating pouch. Finally, these holes were covered with vinyl adhesive to avoid contamination.

\subsection{Fabrication of $3 D \mu P A D s$}

The 3D devices were fabricated by assembling layers of plastic, patterned filter paper, and double-sided adhesive tape in an alternate stacking, as depicted in Fig. 2B. 3D $\mu$ PADs differ from 2D by the use of more than one layer of paper to assemble the device allowing the fluid flow through then. To constructed the 3D devices, first, a hole $(r=5.00 \mathrm{~mm})$ was made on the vinyl adhesive sheet to serve as inlet for sample addition. Then, filter paper was cut into a triangular shape with three external spots $(\mathrm{r}=4.00 \mathrm{~mm})$ at the corners, which were connected to one central spot $(r=5.00 \mathrm{~mm})$.

The third layer was composed of a double-sided adhesive tape with three holes $(\mathrm{r}=2.70 \mathrm{~mm})$ aligned to the three external paper spots, Fig. 2B-c. The double-sided tape was used to keep the layers assembled together and simultaneously to drive the sample solution from the first patterned paper to the detection zones. The detection zones were composed of three disks of filter paper $(\mathrm{r}=4.00 \mathrm{~mm})$, which were previously modified with reagents for colorimetric bioassays after cutting. Each paper disk was positioned matching the holes of the double-side tape. Finally, the 3D device was closed with the vinyl adhesive and kept under pressure for a few seconds to totally seal the whole system (total dimensions of $20.66 \mathrm{~mm} \times 20.66 \mathrm{~mm}$ ). In this case, the lamination pouch is not used since the chemical reagents were previously added to the paper disks and will not supported the heating step required to seal

A - 2D $\mu$ PADs fabrication schematic

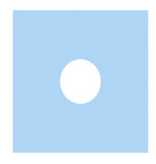

a - Vinyl Adhesive

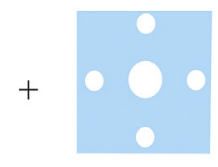

b - Pouch Lamination

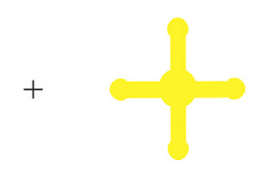

c- Whatman Paper

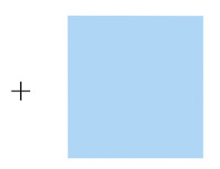

d - Laser Print Sheet
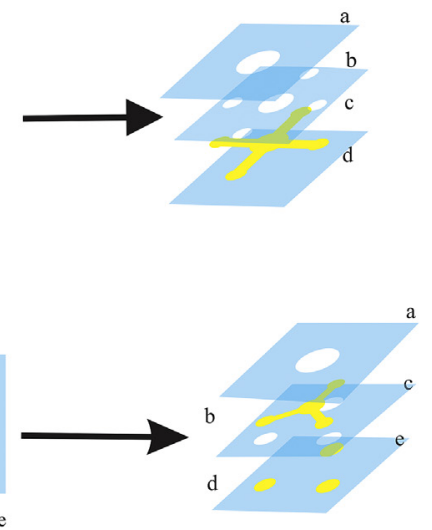

B - 3D $\mu$ PADs fabrication schematic

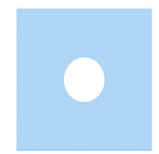

a - Vinyl Adhesive

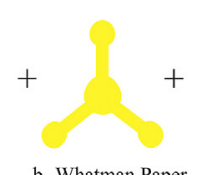

b -Whatman Paper

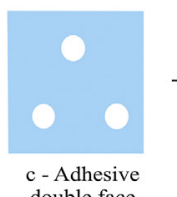

double face

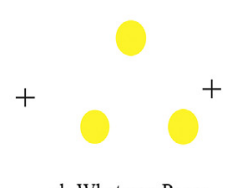

d-Whatman Paper

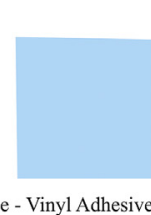

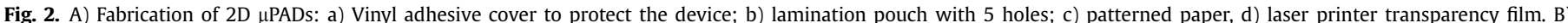

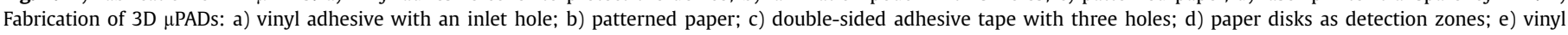
adhesive to seal the device. 


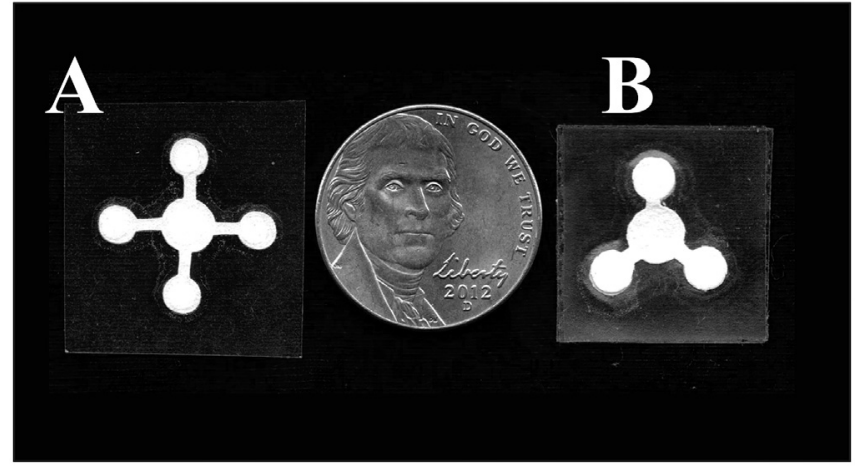

Fig. 3. Pictures of the $2 \mathrm{D}(\mathrm{A})$ and $3 \mathrm{D}(\mathrm{B}) \mu \mathrm{PADs}$.

the microfluidic device. Fig. 3 shows typical pictures of the 2D and 3D $\mu$ PADs developed in this work.
The cross section of the 2D and 3D $\mu$ PADs was analyzed by optical microscopy. Fig. 4 shows cross-sectional pictures of both $\mu$ PADs. For better visualization of the layers, the filter papers were colored with yellow and red dyes in the 2D and 3D devices, respectively, before assembling the $\mu$ PADs. The cross-sectional view of the 2D device (Fig. 4A) shows: a) laminating pouch, b) paper, and c) polyester sheet (laser printer transparency film). The structure of the paper was kept unchanged and all layers were closely attached to each other. During the heat pressing process, the thin polyethylene (PE) film of the lamination pouch melted up, resulting in an effective attachment to the paper and polyester sheet, consequently forming the $2 \mathrm{D}$ device. The PE film was sufficiently thin to not penetrate the paper layer during the heating process. In Fig. 4B, the cross-sectional view of the 3D $\mu$ PAD revealed different layers: $a$ ) vinyl adhesive layer; b) paper detection zone (red color); c) doublesided tape layer; d) loading paper (yellow color), and e) vinyl adhesive layer. In Fig. 4B can also be seen that both paper layers are closed attached beneath the double-side tape layer, allowing the

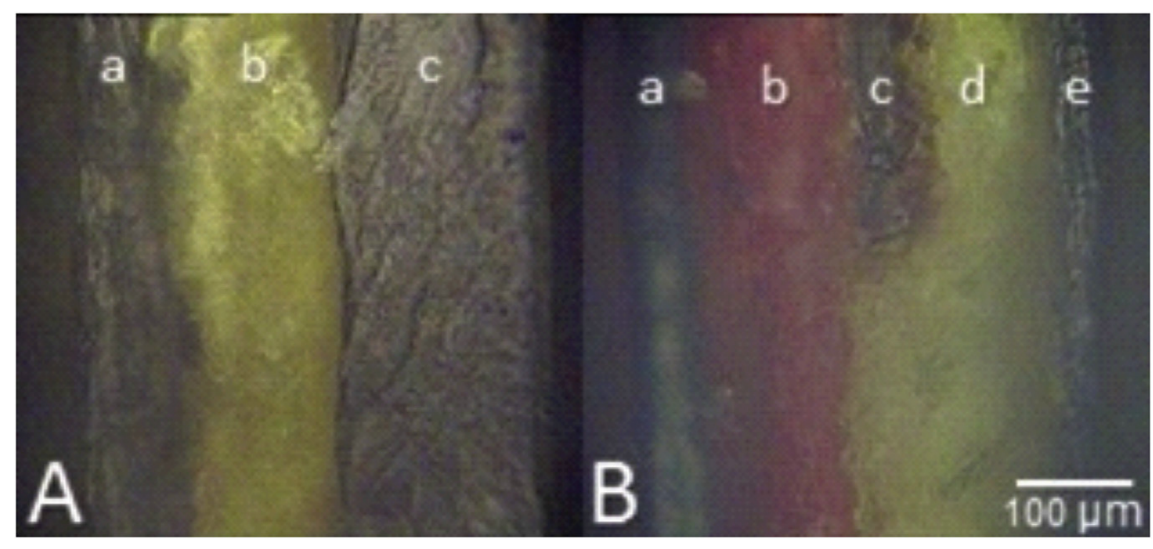

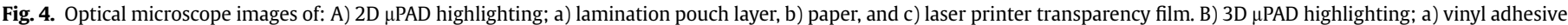
layer, b) detection zone (disk paper), c) loading paper, and d) vinyl adhesive layer. The circle shows the double-sided tape well attached to both paper layers.
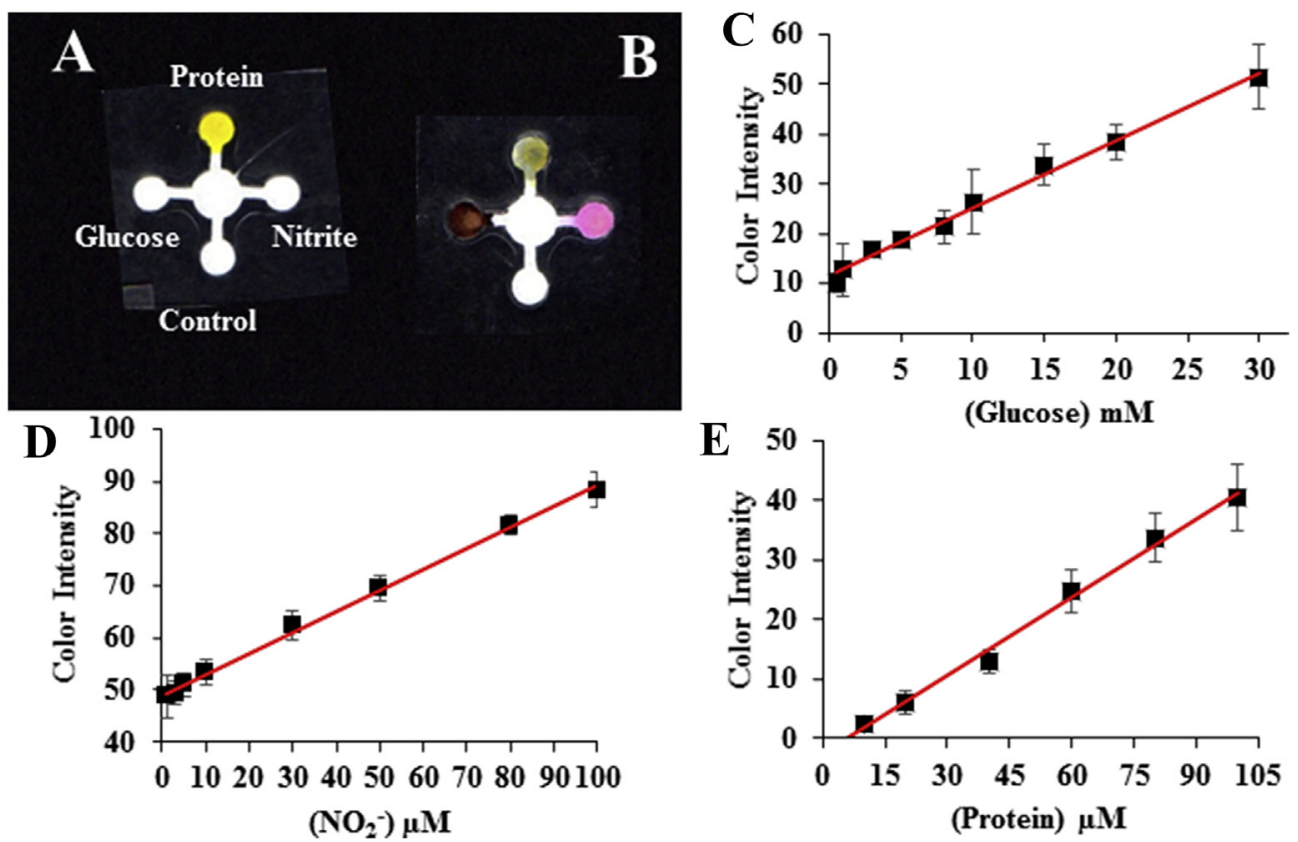

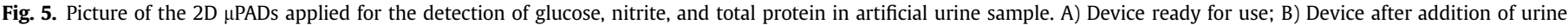

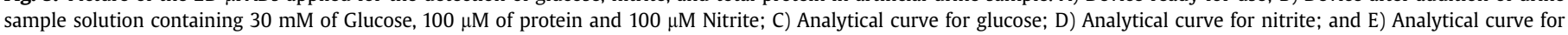
protein. 
solution to flow by capillary effect from the loading paper to the detection zone at the disk paper.

\subsection{Colorimetric detection of glucose, nitrite, and protein in simulated samples}

The feasibility of the $\mu$ PADs developed in this work was demonstrated by the colorimetric detection of three analytes of clinical interest, namely, glucose, nitrite, and total protein, in two different simulated samples (blood serum and urine). These analytes are important parameters for diagnosis and clinical monitoring of health conditions. The blood glucose level is used as a clinical indicator of diabetes [37] and its presence in urine represents a more serious condition because it can indicates glycosuria [38]. The proteins found in urine are mostly related to albumin, whereas the blood proteins are mostly ascribed to albumin and globulin. Elevated levels of protein in urine could be related to temporary health conditions, such as pregnancy [39], stress [40], infections [41], or heavy exercises [42]. High level of total protein in blood could be related to kidney or liver diseases [43]. A high level of nitrite in urine could be associated with urinary tract infection, whereas in blood, nitrite levels are correlated to the nitric oxide (NO), which is a molecule important in a variety biological processes including synaptic plasticity in the central nervous system, central blood pressure regulation, and smooth muscle relaxation [44]. The nitrite assay is based on a colorimetric reaction using Griess reagent [45].

\subsection{1. $2 D \mu P A D$ application in urine sample}

A sample of $8 \mu \mathrm{L}$ of urine spiked with the target analytes at different concentrations was added to the inlet of the 2D $\mu$ PAD. After reacting time of $15 \mathrm{~min}$, the device was photographed and the color generated in each spot was quantified using a photo-editing software. Fig. 5 presents a picture of the 2D $\mu$ PAD without (Fig. 5A) and after addition of urine sample containing $30 \mathrm{mM}$, glucose, protein, and nitrite, respectively (Fig. 5B).

The colorimetric glucose test showed excellent performance, with a linear concentration range of $1-30 \mathrm{mM}$, correlation coefficient $\left(\mathrm{r}^{2}\right)$ of 0.9913 and detection limit (LOD) of $0.44 \mathrm{mM}$. The total protein detection presented a linear response in the interval of $10-100 \mu \mathrm{M}$ with $\mathrm{r}^{2}$ of 0.9939 and LOD of $5.19 \mu \mathrm{M}$. For nitrite, a linear response between 5 and $100 \mu \mathrm{M}$ with $\mathrm{r}^{2}$ of 0.9976 and LOD of $2.34 \mu \mathrm{M}$ was obtained. The analytical curves for glucose, protein, and nitrite are presented in Fig. 5C-E.

The colorimetric biochemical assays for glucose, protein, and nitrite in urine samples performed with the 2D $\mu$ PAD showed excellent analytical performances with good linear concentration ranges and detection limits lower than those reported in literature (see Table S1 in SI). The 2D device presented relative standard deviation (RSD) lower than $8 \%$, as can be observed in Table S1 in SI, which indicates a good reproducibility in the manufacturing technique proposed in this work. These results indicated that the thermal step applied to seal the device did not affect the analytical performance of the $2 \mathrm{D} \mu \mathrm{PADs}$.

\subsection{2. $3 D \mu P A D$ application in blood serum}

In the inlet of $3 \mathrm{D} \mu \mathrm{PAD} 10 \mu \mathrm{L}$ of blood serum sample with the target analytes at different concentrations was added. This device showed a linear response in the concentration range of $1-30 \mathrm{mM}$ with $\mathrm{r}^{2}$ of 0.9981 and LOD of $0.54 \mathrm{mM}$. The analytical response for total protein was linear in the concentration interval of 3-100 $\mu \mathrm{M}$ with $\mathrm{r}^{2}$ of 0.9941 , and LOD of $1.26 \mu \mathrm{M}$. For nitrite, the 3D device showed colorimetric responses in the concentration range of 5-600 $\mu \mathrm{M}$ with $\mathrm{r}^{2}=0.9926$ and LOD of $4.35 \mu \mathrm{M}$. The 3D $\mu$ PAD also presented good reproducibility for these analytes with RSD lower than 6\% (see Table S1 in SI).

Fig. 6 displays the pictures of the 3D $\mu \mathrm{PAD}$ applied for detecting the analytes in blood serum, before and after the sample additions, and their respective analytical curves.

The 3D $\mu$ PAD developed represents a new design compare to previously devices reported. The assays using the $3 \mathrm{D}$ device presented excellent analytical performances, including LODs and linear ranges better than those obtained with the 2D device. The best performance of the 3D device can be related to the use of filter
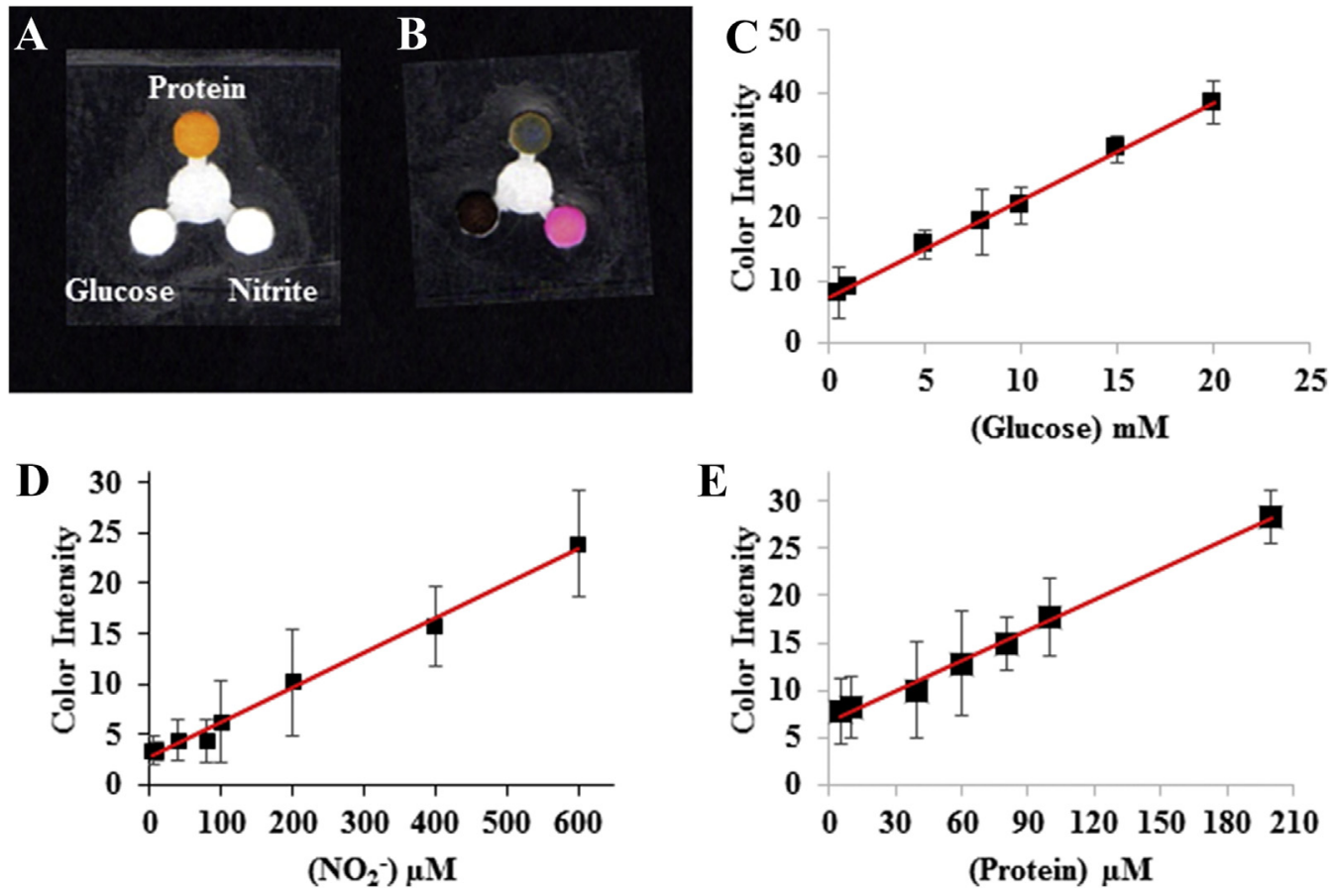

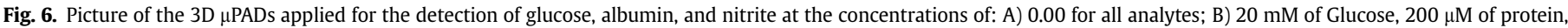
and $600 \mu \mathrm{M}$ Nitrite; C) Analytical curve for glucose; D) Analytical curve for nitrite; and E) Analytical curve for protein. 
paper disks as detection zones. The use of paper disks in the 3D design allowed a better distribution of the sample solution from loading paper to the center of the detection paper disk, thereby forming of well-defined colored spots. Furthermore, the use of a thinner double-sided tape to attach the layers of loading and detection papers allowed the sample solution to flow between them easily without the necessity of filling the gap with cellulose powder or paraffin wax as previously reported $[14,46,47]$ or using a metallic housing firmly attached with screws, as employed in 3D origami-based devices [48].

The analytical performance showed by the 2D and 3D $\mu$ PADs for glucose quantification was proved to be enhanced in terms of LODs over many others reported in literature (see Table S2 in SI) and with good reproducible. The new method proposed here, that includes to cut soft and inexpensive filter paper, to produce 2D and 3D $\mu$ PADs can be easily extended to other colorimetric detection methods including immunoassay applications [49]. Both devices are lightweight, flexible (see Fig. S2), fully protected against contamination, and can be easily handled. In the laboratory scale, a letter size sheet $(215.9 \times 297.4 \mathrm{~mm})$ allowed to construct $962 \mathrm{D}$ and 110 3D devices (Fig. S3) with a cost of approximately $\$ 0.002$ for each device. Compare to $\mu$ PADs built using wax printer, the devices proposed herein need fewer steps to be constructed since after printing the wax, a second step is required that comprises heating the paper for melting the wax and to penetrate in the paper forming a hydrophobic barrier. In this step, the molten wax spreading non-uniformly in the paper in such way that the thickness of the microfluidic channel obtained is lower than that of the original design. With the cutter printer the exact pattern designed can be easily obtained in just one step. In the same way, in our 3D $\mu \mathrm{PADs}$ approach there are no gap between adjacent paper layers and do not require any hydrophilic material to fill the gap as previously reported, that increases the number of steps and the cost to construct the devices.

\section{Conclusions}

This study describes a simple, easily, and cost-effective method to fabricate completely sealed 2D and 3D $\mu$ PADs using filter papers and an inexpensive home cutter printer. We demonstrated that soft filter paper can be easily cut in any desirable pattern and size without tearing. The design of the $3 \mathrm{D} \mu \mathrm{PAD}$ proposed has the advantage of keeping the paper layers closely attached allowing the solution easily flow eliminating the needed of hydrophilic materials filling the gap between the paper layers. The new method proposed brings significant improvements on the development of $\mu$ PADs that can be easily manufactured in large scale and are highly suitable for rapid diagnosis, especially in developing countries.

\section{Acknowledgements}

The authors thank the Fundação de Amparo à Pesquisa do Estado de São Paulo (FAPESP - Proc. No 2015/19890-1), and Conselho Nacional de Desenvolvimento Científico e Tecnológico (CNPq Proc. No 427727/2016-2 and 140484/2013-2), for all financial support.

\section{Appendix A. Supplementary data}

Supplementary data related to this article can be found at http:// dx.doi.org/10.1016/j.aca.2017.01.002.

\section{References}

[1] E. Evans, E.F. Moreira Gabriel, T.E. Benavidez, W.K. Tomazelli Coltro,
C.D. Garcia, Modification of microfluidic paper-based devices with silica nanoparticles, Analyst 139 (2014) 5560-5567.

[2] A.K. Yetisen, M.S. Akram, C.R. Lowe, Paper-based microfluidic point-of-care diagnostic devices, Lab Chip 13 (2013) 2210.

[3] A.W. Martinez, S.T. Phillips, G.M. Whitesides, E. Carrilho, Diagnostics for the developing world: microfluidic paper-based analytical devices, Anal. Chem. 82 (2010) 3-10.

[4] A.W.W. Martinez, S.T.T. Phillips, M.J.J. Butte, G.M.M. Whitesides, Patterned paper as a platform for inexpensive, low-volume, portable bioassays, Angew. Chem. Int. Ed. 46 (2007) 1318-1320.

[5] C. Rivet, H. Lee, A. Hirsch, S. Hamilton, H. Lu, Microfluidics for medical diagnostics and biosensors, Chem. Eng. Sci. 66 (2011) 1490-1507.

[6] K. Macek, H. Bečvářová, Papers, ready-for-use plates, and flexible sheets for chromatography, Chromatogr. Rev. 15 (1971) 1-28.

[7] R. Pelton, Bioactive paper provides a low-cost platform for diagnostics, TrAC Trends Anal. Chem. 28 (2009) 925-942.

[8] L. Ge, S. Wang, X. Song, S. Ge, J. Yu, 3D Origami-based multifunction-integrated immunodevice: low-cost and multiplexed sandwich chemiluminescence immunoassay on microfluidic paper-based analytical device, Lab Chip 12 (2012) 3150.

[9] E. Carrilho, A.W. Martinez, G.M. Whitesides, Understanding wax printing: a simple micropatterning process for paper-based microfluidics, Anal. Chem. 81 (2009) 7091-7095.

[10] S.A. Klasner, A.K. Price, K.W. Hoeman, R.S. Wilson, K.J. Bell, C.T. Culbertson, Paper-based microfluidic devices for analysis of clinically relevant analytes present in urine and saliva, Anal. Bioanal. Chem. 397 (2010) 1821-1829.

[11] W. Dungchai, O. Chailapakul, C.S. Henry, A low-cost, simple, and rapid fabrication method for paper-based microfluidics using wax screen-printing, Analyst 136 (2011) 77-82.

[12] Y. Sameenoi, P. Panymeesamer, N. Supalakorn, K. Koehler, O. Chailapakul, C.S. Henry, et al., Microfluidic paper-based analytical device for aerosol oxidative activity, Environ. Sci. Technol. 47 (2013) 932-940.

[13] W. Wang, W.-Y. Wu, J.-J. Zhu, Tree-shaped paper strip for semiquantitative colorimetric detection of protein with self-calibration, J. Chromatogr. A 1217 (2010) 3896-3899.

[14] A.W. Martinez, S.T. Phillips, Z. Nie, C.-M. Cheng, E. Carrilho, B.J. Wiley, et al., Programmable diagnostic devices made from paper and tape, Lab Chip 10 (2010) 2499.

[15] Z. Nie, C.A. Nijhuis, J. Gong, X. Chen, A. Kumachev, A.W. Martinez, et al., Electrochemical sensing in paper-based microfluidic devices, Lab Chip 10 (2010) 477-483.

[16] C. Li, K. Vandenberg, S. Prabhulkar, X. Zhu, L. Schneper, K. Methee, et al., Paper based point-of-care testing disc for multiplex whole cell bacteria analysis, Biosens. Bioelectron 26 (2011) 4342-4348.

[17] R.F. Carvalhal, M. Simão Kfouri, M.H. de Oliveira Piazetta, A.L. Gobbi, L.T. Kubota, Electrochemical detection in a paper-based separation device, Anal. Chem. 82 (2010) 1162-1165.

[18] J.L. Delaney, C.F. Hogan, J. Tian, W. Shen, Electrogenerated chemiluminescence detection in paper-based microfluidic sensors, Anal. Chem. 83 (2011) 1300-1306.

[19] J. Yu, L. Ge, J. Huang, S. Wang, S. Ge, Microfluidic paper-based chemiluminescence biosensor for simultaneous determination of glucose and uric acid, Lab Chip 11 (2011) 1286.

[20] H.T. Mitchell, I.C. Noxon, C.A. Chaplan, S.J. Carlton, C.H. Liu, K.A. Ganaja, et al., Reagent pencils: a new technique for solvent-free deposition of reagents onto paper-based microfluidic devices, Lab Chip 15 (2015) 2213-2220.

[21] Y. Lu, W. Shi, L. Jiang, J. Qin, B. Lin, Rapid prototyping of paper-based microfluidics with wax for low-cost, portable bioassay, Electrophoresis 30 (2009) 1497-1500.

[22] T. Songjaroen, W. Dungchai, O. Chailapakul, W. Laiwattanapaisal, Novel, simple and low-cost alternative method for fabrication of paper-based microfluidics by wax dipping, Talanta 85 (2011) 2587-2593.

[23] Lab on paper, Lab Chip 8 (2008) 1988-1991.

[24] K. Abe, K. Suzuki, D. Citterio, Inkjet-printed microfluidic multianalyte chemical sensing paper, Anal. Chem. 80 (2008) 6928-6934.

[25] V. Mani, K. Kadimisetty, S. Malla, A.A. Joshi, J.F. Rusling, Paper-based electrochemiluminescent screening for genotoxic activity in the environment, Environ. Sci. Technol. 47 (2013) 1937-1944.

[26] D.A. Bruzewicz, M. Reches, G.M. Whitesides, Low-cost printing of poly(dimethylsiloxane) barriers to define microchannels in paper, Anal. Chem. 80 (2008) 3387-3392.

[27] K.L. Dornelas, N. Dossi, E. Piccin, A simple method for patterning poly(dimethylsiloxane) barriers in paper using contact-printing with low-cost rubber stamps, Anal. Chim. Acta 858 (2015) 82-90.

[28] N. Nuchtavorn, M. Macka, A novel highly flexible, simple, rapid and low-cost fabrication tool for paper-based microfluidic devices ( $\mu$ PADs) using technical drawing pens and in-house formulated aqueous inks, Anal. Chim. Acta 919 (2016) 70-77.

[29] X. Li, J. Tian, T. Nguyen, W. Shen, Paper-based microfluidic devices by plasma treatment, Anal. Chem. 80 (2008) 9131-9134.

[30] P.-K. Kao, C.-C. Hsu, One-step rapid fabrication of paper-based microfluidic devices using fluorocarbon plasma polymerization, Microfluid. Nanofluid. 16 (2014) 811-818.

[31] X. Gong, X. Yi, K. Xiao, S. Li, R. Kodzius, J. Qin, et al., Wax-bonding 3D microfluidic chips, Lab Chip 10 (2010) 2622. 
[32] C.L. Cassano, Z.H. Fan, Laminated paper-based analytical devices (LPAD): fabrication, characterization, and assays, Microfluid. Nanofluid. 15 (2013) $173-181$.

[33] E.M. Fenton, M.R. Mascarenas, G.P. López, S.S. Sibbett, G.P. Lo, S.S. Sibbett, Multiplex lateral-flow test strips fabricated by two-dimensional shaping, ACS Appl. Mater. Interfaces 1 (2009) 124-129.

[34] X. Fang, S. Wei, J. Kong, Paper-based microfluidics with high resolution, cut on a glass fiber membrane for bioassays, Lab Chip 14 (2014) 911.

[35] L. Pires dos Santos Benedetti, V. Bezerra dos Santos, T.A. Silva, E. BenedettiFilho, V.L. Martins, O. Fatibello-Filho, A digital image analysis method for quantification of sulfite in beverages, Anal. Methods 7 (2015) 7568-7573.

[36] A.S. Afonso, C.V. Uliana, D.H. Martucci, R.C. Faria, Simple and rapid fabrication of disposable carbon-based electrochemical cells using an electronic craft cutter for sensor and biosensor applications, Talanta 146 (2016) 381-387.

[37] H. Bo, C. Wang, Q. Gao, H. Qi, C. Zhang, Selective, colorimetric assay of glucose in urine using G-quadruplex-based DNAzymes and 10-acetyl-3,7-dihydroxy phenoxazine, Talanta 108 (2013) 131-135.

[38] M.-Y. Jia, Q.-S. Wu, H. Li, Y. Zhang, Y.-F. Guan, L. Feng, The calibration of cellphone camera-based colorimetric sensor array and its application in the determination of glucose in urine, Biosens. Bioelectron. 74 (2015) 1029-1037.

[39] M.B. Pahwa, S. Seth, A. Khosla, Significance of urine protein/creatinine ratio in pregnancy-induced hypertension, Clin. Chim. Acta 382 (2007) 145-147.

[40] M.F. Carroll, J.L. Temte, Proteinuria in adults: a diagnostic approach, Am. Fam. Physician 62 (2000) 1333-1340.

[41] S. Hwang, J.S. Ham, K.B. Hwang, S.H. Jeong, S.H. Ha, E.H. Koo, et al., Renal artery stenosis presenting with nephrotic-range proteinuria: a case report Kidney Res. Clin. Pract. 35 (2016) 119-122.

[42] M. Peeri, M.-A. Kohanpour, S. Sanavi, H. Matinhomaee, M. Mirsepasi, Effects of different intensities of aerobic exercise on proteinuria in hypoxia and normoxia conditions in young football players, Diálisis Y Traspl. 33 (2012) 84-88.

[43] J.T. Busher, Serum albumin and globulin, in: H.K. Walker, W.D. Hall, J.W. Hurst (Eds.), Clinical Methods, The History, Physical, and Laboratory Examinations, Butterworths, Boston, 1990, pp. 479-499.

[44] U. Förstermann, W.C. Sessa, Nitric oxide synthases: regulation and function, Eur. Heart J. 33 (2012) 829-837.

[45] J. Sun, X. Zhang, M. Broderick, H. Fein, Measurement of nitric oxide production in biological systems by using griess reaction assay, Sensors 3 (2003) 276-284.

[46] A.W. Martinez, S.T. Phillips, G.M. Whitesides, Three-dimensional microfluidic devices fabricated in layered paper and tape, Proc. Natl. Acad. Sci. 105 (2008) 19606-19611.

[47] H. Noh, S.T. Phillips, Metering the capillary-driven flow of fluids in paperbased microfluidic devices, Anal. Chem. 82 (2010) 4181-4187.

[48] H. Liu, R.M. Crooks, Three-dimensional paper microfluidic devices assembled using the principles of origami, J. Am. Chem. Soc. 133 (2011) 17564-17566.

[49] K. Yamada, S. Takaki, N. Komuro, K. Suzuki, D. Citterio, An antibody-free microfluidic paper-based analytical device for the determination of tear fluid lactoferrin by fluorescence sensitization of Tb3+, Analyst 139 (2014) 1637. 